OPEN ACCESS

Edited by:

Hubert Vaudry,

University of Rouen, France

Reviewed by:

Rafael Vazquez-Martinez, University of Cordoba, Spain

Yves Tremblay,

Laval University and Centre Hospitalier

Universitaire de Québec, Canada

${ }^{*}$ Correspondence:

Marta C. Romano,

Departamento de Fisiología, Biofísica y Neurociencias, CINVESTAV del IPN,

Apdo. Postal 14-745,

07360 Mexico city, Mexico mromano@fisio.cinvestav.mx

Specialty section

This article was submitted to Neuroendocrine Science,

a section of the journal

Frontiers in Neuroscience

Received: 11 December 2014 Accepted: 08 June 2015

Published: 30 June 2015

Citation:

Romano MC, Jiménez $P$

Miranda-Brito $C$ and Valdez RA (2015)

Parasites and steroid hormones: corticosteroid and sex steroid

synthesis, their role in the parasite physiology and development.

Front. Neurosci. 9:224. doi: 10.3389/fnins.2015.00224

\section{Parasites and steroid hormones: corticosteroid and sex steroid synthesis, their role in the parasite physiology and development}

\author{
Marta C. Romano ${ }^{*}$, Pedro Jiménez ${ }^{2}$, Carolina Miranda-Brito ${ }^{1}$ and Ricardo A. Valdez ${ }^{1}$ \\ 'Departamento de Fisiología, Biofísica y Neurociencias, CINVESTAV del IPN, Mexico city, Mexico, ${ }^{2}$ Centro de Investigación \\ en Reproducción Animal, CINVESTAV-UAT, Tlaxcala, Mexico
}

In many cases parasites display highly complex life cycles that include the penetration and permanence of the larva or adults within host organs, but even in those that only have one host, reciprocal, intricate interactions occur. Evidence indicates that steroid hormones have an influence on the development and course of parasitic infections. The host gender's susceptibility to infection, and the related differences in the immune response are good examples of the host-parasite interplay. However, the capacity of these organisms to synthesize their own steroidogenic hormones still has more questions than answers. It is now well-known that many parasites synthesize ecdysteroids, but limited information is available on sex steroid and corticosteroid synthesis. This review intends to summarize some of the existing information in the field. In most, but not all parasitosis the host's hormonal environment determines the susceptibility, the course, and severity of parasite infections. In most cases the infection disturbs the host environment, and activates immune responses that end up affecting the endocrine system. Furthermore, sex steroids and corticosteroids may also directly modify the parasite reproduction and molting. Available information indicates that parasites synthesize some steroid hormones, such as ecdysteroids and sex steroids, and the presence and activity of related enzymes have been demonstrated. More recently, the synthesis of corticosteroid-like compounds has been shown in Taenia solium cysticerci and tapeworms, and in Taenia crassiceps WFU cysticerci. In-depth knowledge of the parasite's endocrine properties will contribute to understand their reproduction and reciprocal interactions with the host, and may also help designing tools to combat the infection in some clinical situations.

Keywords: parasites, steroid synthesis, sex steroids, corticosteroids, steroidogenic enzymes, Taenias, cysticerci

\section{Parasites and Host Interplay: Steroid Hormones Influence Parasite Development and Survival}

Progesterone, androgens, and estrogens are present and have critical roles in the vertebrate reproduction and metabolism, but the influence and occurrence of steroid hormones in invertebrates had received less attention (Lafont, 2000) the interplay between the parasite and the host defines the intensity of parasite infections. In many cases, the presence of parasites 
in the host changes its endocrine equilibrium due to the activation of the immune system response, which finally affects the endocrine system through the influence of cytokines and growth factors released by the immune cells. It is now widely accepted that corticosteroids and sex-related hormones influence the immune response (Roberts et al., 2001; Coutinho and Chapman, 2011; Reyes-Hernandez et al., 2013), thereafter any endocrine perturbation initiated by an infection will change the neuroendocrine equilibrium. These hormonal changes resulting from a spontaneous or experimental infection, affect the parasitic charge, the course of the infection and the parasite's survival (Barthelemy et al., 2004). On the other hand, some parasite infections disrupt the host endocrine system, in a noteworthy example, the dromedary bull, Camelus dromedarius, parasitized with Tripanosoma evansi, presents changes in the sexual steroid plasmatic concentrations, as well as in the semen characteristics (Al Qarawi, 2005). Furthermore, the male Fence Lizard (Sceloporus occidentalis) infected with the malarial parasite Plasmodium mexicanum shows several reproductive pathologies, such as fewer courtship and decreased testosterone levels (Dunlap and Schall, 1995) and the Toxoplasma gondee infection enhances testicular steroidogenesis in rats (Lim et al., 2013). Interestingly, it has been shown that the host hormonal environment determines the susceptibility, the course, and severity of many parasite infections, and therefore a clear dichotomy in infection susceptibility between males and females had been observed (Morales-Montor et al., 2004). A rich estrogen environment facilitates Taenia crassiceps cysticerci proliferation, blocking thus the P450-aromatase with fadrozole decreased parasite load (Morales-Montor et al., 2002). Parasites may also alter the host's reproductive behavior (Thompson and Kavaliers, 1994) as had been also shown in Taenia crassiceps ORF infected male mice (Morales et al., 1996).

Steroids and steroid synthesis inhibitors influence the fertility of Schistosoma mansoni in vitro (Morrison et al., 1986) while progesterone, makisterone, and ecdysone increased the length of the larvae Ascaris suum, a roundworm swine parasite (Fleming, 1985), while progesterone, $17 \beta$-estradiol, and testosterone added to the culture medium enhanced the number of Plasmodium falciparum gametocytes (Lingnau et al., 1993). Progesterone, but not testosterone decreased the in vitro molting process of Trichinella spiralis (Hernández-Bello et al., 2011) and T. crassiceps ORF cysticerci cell proliferation was increased by physiological concentrations of testosterone, and $17 \beta$-estradiol added to the culture media (Romano et al., 2003), while high concentrations inhibited its reproduction (Escobedo et al., 2004).

\section{The Role of Corticosteroids in the Host-parasite Interplay}

It is well-known that non-physiological stress situations, such as social isolation, infections, persecution, etc., increase serum corticosteroids levels with the consequent impairment of the immune response. The interplay host-parasite is not the exception, for example social stress caused by female isolation increased blood Typanosoma cruzi infection in the wild mouse Calomys callosus, which showed body weight loss and impaired immune response (Santos et al., 2008), whereas the hypothalamus-pituitary-interregnal axis of rainbow trout was altered by the parasite haemoflagelate Cryptobia infection (Madison et al., 2013). In addition, the infection with Anguillicola novaezelandiae affects cortisol levels in the European Eel (Dangel et al., 2014). Frequently, the host and the parasites are affected in the course of infection, as in Bluegill Sunfish (Lepomis macrochirus) infected with Utterbackia imbecillis, which results in plasma cortisol increment in the host, favoring thus the parasite juvenile metamorphosis (Dubansky et al., 2011). On the other hand, in vivo treatment of rats with cortisol increased the growth rate of the protozoan parasite Toxoplasma gondii in isolated peritoneal macrophages (Wang et al., 2014).

\section{Direct Effects of Glucocorticoids on Parasite Growth}

Aside from the influence of corticosteroids in the course of parasitic infections, it had been shown that these hormones directly influence parasite's growth. For instance, cortisol and dexamethasone increase the in vitro multiplication of the haemoflagellate Cryptobia salmonistica, possible by their interaction with glucocorticoid receptor-like protein ( $\mathrm{Li}$ et al., 2013, 2014). In this regard, dehydroepiandrosterone (DHEA) addition to the culture media decreased, while cortisol increased the in vitro growth and viability of Entamoeba histolytica (Carrero et al., 2006). We had shown that corticosterone and dexamethasone increase the capacity of $T$. crassiceps WFU cysticerci to synthesize androgens and estrogens, hormones that favor the parasite reproduction (Hinojosa et al., 2011).

\section{Parasites Synthesize Steroid Hormones}

\section{Lipids and Steroid Hormones}

Lipids, and particularly cholesterol and their metabolites, are required and synthesized by some parasites. Bansal et al. (2005) reviewed the requirement for lipids, particularly cholesterol, by pathogens like protozoa (Leishmaniosis, Malaria, and Toxoplasmosis). It has recently been stated that cholesterol exerts many of its functions by maintaining a specialized type of membrane domain called "lipid rafts" in a functional state. These domains are rich in cholesterol and sphingolipids and could be involved in signal transduction and in the entry of pathogens to the host cells (Simons and Toomre, 2000).

The incorporation and utilization of arachidonic acid, linoleic acid, 3-sn-phosphatidycholine, tripalmitylglycerol,and cholesterol by adult Schistosoma mansoni was demonstrated by Rumjanek and Simpson (1980). These parasites exchange cholesterol and other metabolites during reproduction (Popiel and Basch, 1986; Silveria et al., 1986), while cholesterol is absorbed by the hydatid cysts of Echinococcus granulosus (Bahr et al., 1979). On the other hand, parasites like Trypanosome cruzi and some species of Leishmania cannot use cholesterol but they synthesize ergosterol and related 
24-alkylated sterols. Furthermore, Leishmania has a strict requirement for ergosterol for their survival and growth (Urbina et al., 2002; Magaraci et al., 2003; Bazin et al., 2006).

\section{Ecdysteroids}

Several parasites synthesize ecdysteroids, steroid hormones that are essential for arthropod molting; the capacity to synthesize these hormones has been used to classify cestodes (de Loof and Huybrechts, 1998). However, the role of these steroids in parasites remains obscure. Ecdysterone production has been reported in a variety of helminth species, among them, the nematodes Dirofilaria immitis, Brugia pahangi, Ascaris suum, and Anisakis simplex, the cestodes: Moniezia expansa, Echinococcus granulosus, and Hymenolepis diminuta and the trematodes: Fasciola hepatica and Schistosoma mansoni (Mendis et al., 1983, 1984; Fleming, 1985; Cleator et al., 1987; Evershed et al., 1987; Mercer et al., 1987a,b, 1990; Foster et al., 1992). In male and female, Dirofilaria immitis, and in female, Ascaris suum ecdysteroids were concentrated in the reproductive system, whereas the presence of ecdysteroids in the eggs of Schistosoma mansoni suggests a regulatory role in embryogenesis (Mercer, 1985). Ecdysteroids have also been found in cestodes like the sheep parasite Moniezia expansa and in the rat tapeworm Hymenolepis diminuta (Mercer et al., 1987b). Ecdysterone can be detected in the sera of infected hosts parasitized by Schistosoma, a trematode that synthesizes and releases the steroid to the host's circulation (Mercer, 1985). Although the role of ecdysteroids during insect metamorphosis had been widely demonstrated, the function of these steroids in parasites remains obscure.

\section{Sex Steroids}

Evidence for the steroidogenic capacity of Schistosoma mansoni was provided years ago by data showing the conversion of steroid precursors to its metabolites by the parasite homogenate (Briggs, 1972). We had shown that cultured Trypanosoma cruzi trypomastigotes synthesize androgens and estrogens from androstenedione and DHEA (Vacchina et al., 2008). We had also investigated the steroidogenic capacity of the cysticerci and tapeworm from Taenia solium and Taenia crassiceps WFU. The adult worm of Taenia solium and crassiceps WFU tapeworms are attached to the host gut with hooks that surrounds their head, and develop reproductive units called proglottids, where testis and ovaries gradually differentiate, and finally contain spermatocytes and infective eggs (Willms et al., 2003). Taenia solium cysticerci is the larval stage of the parasite and is found in the brain or muscle of humans and pigs, whereas Taenia crassiceps WFU cysticerci constitute a useful laboratory model due to their reproduction by budding in the peritoneal cavity of mice. We had been exploring the capacity of cysticerci to synthesize sex steroids in vitro and found that T. solium and T. crassiceps ORF cysticerci transform steroid precursors such as progesterone, dehydroepiandrostenedione, and androstenedione to androgens and estrogens (Gómez et al., 2000; Jiménez et al., 2006; Valdez et al., 2006). Other experiments showed that drugs that block steroidogenic enzymes interfered the in vitro steroid synthesis, suggesting the existence of this enzymes in the parasites (Aceves-Ramos et al., 2013).

Because of the presence of testis and ovaries in tapeworm proglottids, we had investigated the in vitro steroidogenic capacity of experimental Taenia crassiceps WFU and solium tapeworms obtained from the intestine of infected hamsters. Taenia crassiceps WFU tapeworms were incubated in the presence of ${ }^{3} \mathrm{H}$-DHEA and tritiated androstenediol and $17 \beta$-estradiol where recovered from the culture media, which strongly suggest the presence and activity of enzymes from the $\Delta 5$ steroid pathway in these tapeworms (Fernández Presas et al., 2008). Taenia solium tapeworms kept in culture also synthesized sex steroids (Valdez et al., 2014).

\section{Corticosteroids}

Since the above mentioned literature indicated that Taenia solium and Taenia crassiceps cysticerci and tapeworms synthesized sex steroid hormones, we thought these organisms could also synthesize corticosteroids. Thereafter, we had incubated Taenia crassiceps cysticerci in the presence of ${ }^{3} \mathrm{H}$-progesterone and found an important transformation into deoxycorticosterone (Valdez et al., 2012), a steroid that has mineralocorticoid functions in vertebrates and also display some glucocorticoid properties. In addition, the parasites synthesized corticosterone, which was measured by radioimmunoassay in the culture media. More recently, we found corticosteroid-like synthesis in Taenia solium tapeworms (Valdez et al., 2014). It had been shown that in the adrenal reticular zone of mammals an excess of progesterone can be inactivated by $20 \mathrm{HSD}$ activity (Pelletier et al., 2005) and that cortisol and corticosterone can be inactivated by $11 \beta$-hydroxylase in the adrenal glomerulose zone (for a review see Odermatt and Atanasov, 2009) but to our knowledge these enzymes had not been investigated in Taenia solium or crassiceps WFU organisms. Besides their effects on the own parasite development and differentiation, the cysticerci, and tapeworm's steroidogenic capacity might play a role in the permanence of the parasites in muscle and brain tissues and in the host intestine.

\section{Parasites Express Steroidogenic Enzymes}

Some steroidogenic enzymes had been described in parasites. For instance, sterol-sterifying enzymes were found in Toxoplasma gondee, a protozoan incapable of cholesterol de novo synthesis (Lige et al., 2013). The presence of $3 \beta$ hydroxysteroid dehydrogenase in Sarcocystis spp had been shown by immunohistochemistry (Yarim et al., 2004); the enzyme is also present in Taenia solium cysticerci and tapeworms (Fernández Presas et al., 2008). Genes from the cholesterol synthesis pathway have been found and expressed in Giardia intestinalis (Hernandez and Wasserman, 2006). The parasite flatworm Schistosoma japonicum has a type 12 17-HSD that metabolizes estrone to estradiol (Zhou et al., 2009).

We have recently shown that Taenia solium cysticerci express the enzyme $17 \beta$-HSD that belongs to the short 
chain dehydrogenases/reductase family (Aceves-Ramos et al., 2014). Transient transfection of HEK293T cells with Tsol17 $\beta$ HSD-pcDNA3.1 (+) induced expression of Tsol17 $\beta$-HSD that transformed ${ }^{3} \mathrm{H}$-androstenedione into testosterone. In contrast, ${ }^{3} \mathrm{H}$-estrone was not significantly transformed into estradiol. Therefore, Taenia solium cysticerci express a $17 \beta$-HSD that catalyzes the androgen reduction and belongs to the short chain dehydrogenases/reductase (SDR) protein superfamily (AcevesRamos et al., 2014). Recently, a sequence (EmW000624600 which is available at: www.genedb.org/Homepage/Emultilocularis) with an identity of $84 \%$ with Tsol-17ßHSD and a total coverage has been described for E. multilocularis, suggesting the presence of 17 HSD enzymes in this Taeniid family (Tsai et al., 2012). However, the expression level and enzyme activity has not been yet investigated.

\section{References}

Aceves-Ramos, A., de la Torre, P., Hinojosa, L., Ponce, A., García-Villegas, R., Laclette, J. P., et al. (2014). Cloning, characterization and functional expression of Taenia solium 17 beta-hydroxysteroid dehydrogenase. Gen. Comp. Endocrinol. 203, 186-192. doi: 10.1016/j.ygcen.2014.03.021

Aceves-Ramos, A., Valdez, R. A., Gaona, B., Willms, K., and Romano, M. C. (2013). Steroid synthesis by Taenia crassiceps WFU cysticerci is regulated by enzyme inhibitors. Gen. Comp. Endocrinol. 188, 212-217. doi: 10.1016/j.ygcen.2013.03.034

Al Qarawi, A. A. (2005). Infertility in the dromedary bull: a review of causes, relations and implications. Anim. Reprod. Sci. 87, 73-92. doi: 10.1016/j.anireprosci.2004.11.003

Bahr, J. M., Frayha, G. J., and Hajjar, J. J. (1979). Mechanism of cholesterol absorption by the hydatid cysts of Echinococcus granulosus (Cestoda). Comp. Biochem. Physiol. 62A, 485-490. doi: 10.1016/0300-9629(79) 90090-2

Bansal, D., Bahatti, H. S., and Seghal, R. (2005). Role of cholesterol in parasitic infections. Lipid. Health Dis. 9, 4-10. doi: 10.1186/1476-511X-4-10

Barthelemy, M., Gabrion, C., and Petit, G. (2004). Reduction in testosterone concentration and its effect on the reproductive output of chronic malariainfected male mice. Parasitol. Res. 93, 475-481. doi: 10.1007/s00436-0041160-2

Bazin, M. A., Loiseau, P. M., Bories, C., Letoumeux, Y., Rault, S., and EI Kihen, L. (2006). Synthesis of oxysterols and nitrogenous sterols with antileshmania and tripacidal activities. Eur. J. Med. Chem. 41, 1109-1116. doi: 10.1016/j.ejmech.2006.03.033

Briggs, M. H. (1972). Metabolism of steroid hormones by schistosomes. Biochim. Biophys. Acta 280, 480-485. doi: 10.1016/0005-2760(72)90256-1

Carrero, J. C., Cervantes, C., Moreno-Mendoza, N., Saavedra, E., and MoralesMontor, J. (2006). Dehydroepiandrosterone decreases while cortisol increases in vitro growth and viability of Entamoeba histolytica. Microbes Infect. 8, 323-331. doi: 10.1016/j.micinf.2005.06.030

Cleator, M., Delves, C. J., Howells, R. E., and Rees, H. H. (1987). Identity and tissue localization of free and conjugated edcysteroids in adults of Dirofilaria immitis and Ascaris surum. Mol. Biochem. Parasitol. 25, 93-105. doi: 10.1016/01666851(87)90022-3

Coutinho, A. E., and Chapman, K. E. (2011). The anti-inflammatory and immunosuppressive effects of glucocorticoids, recent developments and mechanistic insights. Mol. Cell Endocrinol. 335, 2-13. doi: 10.1016/j.mce.2010.04.005

Dangel, K. C., Keppel, M., Tabujew, K., and Sures, S. (2014). Effects of Anguillicola novaezelandiae on the levels of cortisol and hsp70 in the European Eel. Parasitol. Res. 113, 3817-3822. doi: 10.1007/s00436-014-4049-8

de Loof, A., and Huybrechts, R. (1998). "Insects do not have sex hormones": a myth? Gen. Comp. Endocrinol. 111, 245-260. doi: 10.1006/gcen.1998.7101

\section{Concluding Remarks}

The knowledge of parasite endocrinology will contribute to our understanding of parasite biology and their interactions with the host. Sex steroids and corticosteroids are important hormones for the growth, differentiation, and performance in many species. Therefore, the synthesis of these hormones by parasites themselves may be critical for their own development and viability. In addition, it was shown that the production of steroids by parasites is regulated by corticosteroids and affected by steroidogenic inhibitors, which may be used as tools to combat the infection in some clinical situations. Furthermore, steroid synthesis by parasites may contribute to defend them from the attack of immune cells and therefore facilitate their survival in the host tissues.

Dubansky, B., Whitaker, B., and Galvez, F. (2011). Influence of cortisol on the attachment and metamorphosis of larval Utterbackia imbecillis on Bluegill Sunfish (Lepomis macrochirus). Biol. Bull. 220, 97-106. doi: 10.2307/23046932

Dunlap, D. D., and Schall, J. J. (1995). Hormonal alterations and reproduction inhibition in male fence lizards (Sceloporus occidentals) infected with the malarial parasite Plasmodium mexicanum. Physiol. Zool. 68, 608-621.

Escobedo, G., Larralde, C., Chavarria, A., Cerbón, M. A., and Morales-Montor, J. (2004). Molecular mechanisms involved in the differential effects of sex steroids on the reproduction and infectivity of Taenia crassiceps. J. Parasitol. 6, 1235-1244. doi: 10.1645/GE-297R

Evershed, R. P., Mercer, J. G., and Rees, H. H. (1987). Capillary gas chromatography-mass spectrometry of ecdysteroids. J. Chromatogr. 390, 357-369. doi: 10.1016/S0021-9673(01)94387-0

Hernández-Bello, R., Ramirez-Nieto, R., Muñiz-Hernández, S., Nava-Castro, K., Pavón, L., Sánchez-Acosta, A. G., et al. (2011). Sex steroids effects on the molting process of the helminth human parasite Trichinella spiralis. J. Biomed. Biotechnol. 2011:625380. doi: 10.1155/2011/625380

Fernández Presas, A. M., Willms, K., and Romano, M. C. (2008). The key steroidogenic enzyme 3ß-hydroxysteroid dehydrogenase is present in the strobilae and larvae of Taenia solium and T. crassiceps WFU strain. Parasitol. Res. 103, 847-852. doi: 10.1007/s00436-008-1066-5

Fleming, M. W. (1985). Ascaris suum; role of ecdysteroids in molting. Exp. Parasitol 60, 207-210. doi: 10.1016/0014-4894(85)90024-4

Foster, J. M., Mercer, J. G., and Rees, H. H. (1992). Analysis of ecdysteroids in the trematodes, Schistosoma mansoni and Fasciola hepatica. Trop. Med. Parasitol. 43, 239-244.

Gómez, Y., Valdez, R. A., Larralde, C., and Romano, M. C. (2000). Sex steroids and parasitism. Taenia crassiceps cysticercus metabolizes exogenous androstenedione to testosterone in vitro. J. Steroid. Biochem. Mol. Biol. 74, 143-147. doi: 10.1016/S0960-0760(00)00099-6

Hernandez, P. C., and Wasserman, M. (2006). Do genes from the cholesterol pathway exist and express in Giardia intestinalis? Parsitol. Res. 98, 194-199. doi: 10.1007/s00436-005-0039-1

Hinojosa, L., Valdez, R. A., Salvador, V., Rodriguez, A. G., Willms, K., and Romano, M. C. (2011). The effect of corticosteroids on sex steroid synthesis in cultured Taenia crassiceps Wake Forest University (WFU) cysticerci. J. Helminthol. 86, 465-469. doi: 10.1017/S0022149X11000708

Jiménez, P., Valdéz, R. A., and Romano, M. C. (2006). Metabolism of steroid hormones by Taenia solium and Taenia crassiceps cysticerci. J. Steroid Biochem. Mol. 99, 203-208. doi: 10.1016/j.jsbmb.2006.01.002

Lafont, R. (2000). The endocrinology of invertebrates. Ecotoxicology 9, 41-57 doi: 10.1023/A:1008912127592

Li, M., Leatherland, J. F., and Woo, P. T. K. (2013). Cortisol and dexamethasone increase the in vitro multiplication of the haemoflagellate Cryptobia salmonistica, possible by interaction with glucocorticoid receptor-like protein. Int. J. Parasitol. 43, 353-360. doi: 10.1016/j.ijpara.2012.11.009 
Li, M., Patrick, T. K., and Woo, P. T. K. (2014). Glucocorticoid receptors on and in a unicellular organism, Cryptobia salmonistica. Int. J. Parasitol. 44, 205-210. doi: 10.1016/j.ijpara.2013.10.006

Lige, B., Sampels, V., and Coppens, I. (2013). Characterization of a second sterolesterfying enzyme in Toxoplama highlights the importance of cholesterol storage pathways for the parasite. Mol. Microbiol. 87, 951-967. doi: $10.1111 / \mathrm{mmi} .12142$

Lim, A., Kumar, V., Hari Dass, S. A., and Vyas, A. (2013). Toxoplasma gondee infection enhances testicular steroidogenesis in rats. Mol. Ecol. 22, 102-110. doi: $10.1111 /$ mec. 12042

Lingnau, A., Margos, G., Maier, W. A., and Seitz, H. M. (1993). The effects of hormones on the gametocytogenesis of Plasmodium falciparum in vitro. Appl. Parasitol. 34, 153-160.

Madison, B. N., Woo, P. T., and Bernier, N. J. (2013). Duress without stress: cryptobia infection results in HPI disfunction in rainbow trout. J. Endocrinol. 218, 287-297. doi: 10.1530/JOE-13-0155

Magaraci, F., Jiménez, C. J., Rodrigues, C., Rodrígues, J. C., Braga, M. V., Yardley, V., et al. (2003). Azasterols as inhibitors of sterol 24-methyltransferase in Leishmania species and Tryposoma cruzi. J. Med. Chem. 46, 4714-4727. doi: 10.1021/jm021114j

Mendis, A. H., Rees, H. H., and Gooswin, T. W. (1984). The occurrence of ecdysteroid in the cestode. Monieza expansa. Mol. Biochem. Parasitol. 10, 123-138. doi: 10.1016/0166-6851(84)90001-X

Mendis, A. H., Rose, M. E., Rees, H. H., and Goodwin, T. W. (1983). Ecdysteroids in adults of the nematode, Dirofilaria immitis. Mol. Biochem. Parasitol. 9, 209-226. doi: 10.1016/0166-6851(83)90098-1

Mercer, J. G. (1985). Developmental hormones in parasitic helminths. Parasitol. Today $1,96-100$.

Mercer, J. G., Barker, G. C., Howells, R. E., and Rees, H. H. (1990). Investigation of ecdysteroid excretion by adult Dirofilaria immitis and Brugia pahangi. Mol. Biochem. Parasitol. 38, 89-95. doi: 10.1016/0166-6851(90)90208-4

Mercer, J. G., Munn, A. E., and Rees, H. H. (1987a). Echinococcus granulosus: occurrence of ecdcysteroids in protoscoleces and hydatid cyst fluid. Mol. Biochem. Parasitol. 24, 203-214. doi: 10.1016/0166-6851(87)90107-1

Mercer, J. G., Munn, A. E., and Rees, H. H. (1987b). Analysis of ecdsysteroids in different developmental stages of Hymenolepis diminuta. Mol. Biochem. Parasitol. 25, 61-71. doi: 10.1016/0166-6851(87)90019-3

Morales, J., Larralde, C., Arteaga, M., Govezensky, T., Romano, M. C., and Moralí, G. (1996). Inhibition of sexual behavior in male mice infected with Taenia Crassiceps cysticerci. J. Parasitol. 82, 689-693. doi: 10.2307/ 3283875

Morales-Montor, J., Chavarria, A., de Leon, M. A., del Castillo, L. I., Escobedo, E. G., Sanchez, E. N., et al. (2004). Host gender in parasitic infections of mammals: an evaluation of the female host supremacy paradigm. J. Parasitol. 90, 531-546. doi: $10.1645 /$ GE-113R3

Morales-Montor, J., Hallal-Caballeros, C., Romano, M. C., and Damian, R. T. (2002). Inhibition of P450-aromatase prevents feminization and induces protection during cisticercosis. Int. J. Parasitol. 32, 1379-1387. doi: 10.1016/S0020-7519(02)00130-3

Morrison, D. D., Vandee Waa, E., and Bennett, J. L. (1986). Effects of steroids and steroid synthesis inhibitors on fecundity of Schistosoma mansoni in vitro. J. Chem. Ecol. 12, 1901-1908. doi: 10.1007/BF01022391

Odermatt, A., and Atanasov, A. G. (2009). Mineralocorticoid receptors: emerging complexity and functional diversity. Steroids 74, 163-171. doi: 10.1016/j.steroids.2008.10.010

Pelletier, G., Luu-The, V., Li, S., and Labrie, F. (2005). Localization of type 5 17ßhydroxysteroid dehydrogenase mRNA in mouse tissues as studied by in situ hybridization. Cell Tissue Res. 320, 393-398. doi: 10.1007/s00441-005-1105-9

Popiel, I., and Basch, P. F. (1986). Schistosoma mansoni colesterol uptake by paired and unpaired worms. Exp. Parasitol. 61, 343-347. doi: 10.1016/00144894(86)90189-X

Reyes-Hernandez, J. L., Leung, G., and McKay, D. M. (2013). Cestode regulation of inflammation and inflammatory research. Int. J. Parasitol. 43, 233-243. doi: 10.1016/j.ijpara.2012.09.005

Roberts, C. W., Walker, W., and Alexander, J. (2001). Sex-associated hormones and immunity of protozoan parasites. Clin. Microbiol. Rev. 14, 476-488. doi: 10.1128/CMR.14.3.476-488.2001
Romano, M. C., Valdez, R. A., Cartas, A. L., Gómez, Y., and Larralde, C. (2003). Steroid hormone production by parasites: the case of Taenia crassiceps and Taenia solium cysticerci. J. Steroid Biochem. Mol. Biol. 85, 221-225. doi: 10.1016/S0960-0760(03)00233-4

Rumjanek, F. D., and Simpson, A. J. G. (1980). The incorporation and utilization of radiolabelled lipids by adult Schistosoma mansoni in vitro. Mol. Biochem. Parasitol. 1, 31-44.

Santos, C. D., Toldo, M. P., Levy, A. M., and Prado, J. C. Jr. (2008). Trypanosoma cruzi: effects of social stress in Calomys callosus a natural reservoir of infection. Exp. Parasitol. 119, 197-201. doi: 10.1016/j.exppara.2008.01.011

Silveria, A. M., Friche, A. A., and Rumjanek, F. D. (1986). Transfer of (14C) cholesterol and its metabolities between adult male and female worms of schistosoma mansoni. Comp. Biochem. Physiol. B 85, 851-857. doi: 10.1016/0305-0491(86)90186-0

Simons, K., and Toomre, D. (2000). Lipids rafts signal transduction. Nat. Rev. 1, 31-39. doi: $10.1038 / 35036052$

Thompson, S. N., and Kavaliers, M. (1994). Physiological basis for parasite induced alterations of host behavior. Parasitology 109, S119-S138. doi: $10.1017 /$ S0031182000085139

Tsai, I. J., Zarowiecki, M., Holroyd, N., Garciarrubio, A., Sanchez-Flores, A., Brooks, K. L., et al. (2012). The genomes of four tapeworm species reveal adaptations to parasitism. Nature 496, 57-63. doi: 10.1038/nature 12031

Urbina, J. A., Conception, J. L., Rangel, S., Visval, G., and Lira, R. (2002). Mol. Squalene synthase as a chemotherapeutic target in Tripsosoma cruzi and Leishmania mexicana. Biochem. Parasitol. 125, 35-45. doi: 10.1016/S01666851(02)00206-2

Vacchina, P., Valdez, R. A., Revelli, S., and Romano, M. C. (2008). Steroidogenic capacity of Trypanosome cruzi trypomastigotes. J. Steroid Biochem. Mol. Biol. 111, 282-286. doi: 10.1016/j.jsbmb.2008.06.016

Valdez, R. A., Hinojosa, L., Gómez, Y., Willms, K., and Romano, M. C. (2012). Taenia crassiceps WFU cysticerci synthesize corticosteroids in vitro: metyrapone regulates the production. Comp. Endocrinol. 176, 409-414. doi: 10.1016/j.ygcen.2012.01.015

Valdez, R. A., Jiménez, P., Cartas, A. L., Gómez, Y., and Romano, M. C. (2006). Taenia solium cysticerci synthesize androgens and estrogens in vitro. Parasitol. Res. 98, 472-476. doi: 10.1007/s00436-005-0095-6

Valdez, R. A., Jiménez, P., Fernández-Presas, A. M., Aguilar, L., Willms, K., and Romano, M. C. (2014). Taenia solium tapeworms synthesize corticosteroids and sex steroids in vitro. Gen. Comp. Endocrinol. 205, 62-67. doi: 10.1016/j.ygcen.2014.04.014

Wang, T., Gao, J.-M., Yi, S. Q., Geng, G. Q., Gao, X. J., Shen, J. L., et al. (2014). The growth rate of Toxoplasma gondii, a protozoan parasite, increased in the peritoneal macrophages of rats treated with glucocorticoids in vivo. Parasitol. Res. 113, 351-358. doi: 10.1007/s00436-013-3661-3

Willms, K., Caro, J. A., and Robert, L. (2003). Ultrastructure of spermatogonia and spermatocyte lobules in Taenia solium strobilac (Cestoda, Cyclophyllidea, Taeniidae) from golden hamsters. Parasitol. Res. 90, 479-488. doi: 10.1007/s00436-003-0897-3

Yarim, M., Yildiz, K., and Kabakci, N. (2004). Immunohistochemical localization of 3ß-hydroxysteroid dehydrogenase in Sarcocystis sp. Parasitol. Res. 93, 457-460. doi: 10.1007/s00436-004-1152-2

Zhou, Y., Zheng, H., Chen, Y., Zhang, L., Wang, K., Guo, J., Huang, Z., et al. (2009). The Schistosoma japonicum genome reveals features of host-parasite interplay. Nature 460, 345-351. doi: 10.1038/nature08140

Conflict of Interest Statement: The authors declare that the research was conducted in the absence of any commercial or financial relationships that could be construed as a potential conflict of interest.

Copyright (๑) 2015 Romano, Jiménez, Miranda-Brito and Valdez. This is an openaccess article distributed under the terms of the Creative Commons Attribution License (CC BY). The use, distribution or reproduction in other forums is permitted, provided the original author(s) or licensor are credited and that the original publication in this journal is cited, in accordance with accepted academic practice. No use, distribution or reproduction is permitted which does not comply with these terms. 